\section{Effect of Aspirin on Reducing Thrombosis of Arteriovenous Fistulae in Uremic Patients Undergoing Hemodialysis}

Sir,

Arteriovenous fistula (AVF) is the main permanent vascular access in uremic patients with maintenance hemodialysis. ${ }^{1}$ Mature arteriovenous fistula can not only provide sufficient blood flow for hemodialysis patients, but also has long use time and few complications. Thrombosis of arteriovenous fistulae is the most important cause for dysfunction of arteriovenous fistula. ${ }^{2}$ Predicting and preventing internal fistula thrombosis has always been an important issue. Anti-platelet aggregation agents have been used as a routine treatment for thrombotic diseases. Aspirin is currently recognised as a safe, effective, and anti-platelet aggregation drug for long-term use. Studies have shown that low-dose oral administration of aspirin can inhibit platelet activity in uremic patients with maintenance hemodialysis. ${ }^{3}$ The authors found that aspirin also has a certain effect on the prevention of internal fistula thrombosis.

It was an experimental study carried out from August 2016 to June 2017. This study was approved by the Hospital Ethics Committee. A total of 100 uremic patients with routine hemodialysis were enrolled in the study. Patients with complicated vascular stenosis, poor arterial blood supply, skin infection in the forearm surgery area, extensive burns, and severe heart failure were excluded. Using the random number table, the patients were randomly divided into control group and intervention group, 50 cases in each group. In the control group, the blood pressure was controlled at $130-160 / 80-100 \mathrm{mmHg}(1 \mathrm{mmHg}=0.033 \mathrm{kPa})$. Bicarbonate dialysate was dialysed three times a week for 4 hours/time. Unfractionated heparin was used for anticoagulation, and its dosage was not more than $12500 \mathrm{U} / \mathrm{d}$, dosage per hours was not more than $2500 \mathrm{U}$, continuous use for 3 days. Patients in the intervention group additionally received oral aspirin administration of $100 \mathrm{mg}$ daily for 12 months. Both groups did not take any other antiplatelet agent during the treatment period. All patients were asked for 3-monthly follow-up for 12 months. Data was analysed in SPSS version 25. Enumeration data was expressed in $n(\%)$ and Chi-square test was applied to compare it. Results were considered significant at $\mathrm{p}<0.05$.

Among 100 cases, $52(52.0 \%)$ were males and 48 (48.0\%) were females, aged 50-70 (62.26 \pm 4.53$)$ years. After 12 months of follow-up, the control group showed murmur and a decrease in vascular tremor. Ten cases $(20.0 \%)$ of internal fistula thrombosis were confirmed by ultrasound. After administration of $400,000 \mathrm{U} / \mathrm{d}$ urokinase thrombolysis and $5000 \mathrm{U} / \mathrm{d}$ low molecular weight heparin calcium for 3 days in the hospital, there were 6 patients $(12.0 \%)$ without vascular murmur and tremor. Ultrasound confirmed their internal fistula dysfunction. These patients were then given internal jugular vein catheter dialysis, and underwent elective surgery to re-establish the arteriovenous fistula for treatment.

In the intervention group, there were 3 cases $(6.0 \%)$ with murmur and a decrease in vascular tremor; and internal fistula thrombosis was confirmed by ultrasound. After treatment with $400,000 \mathrm{U} / \mathrm{d}$ urokinase thrombolysis and $5000 \mathrm{U} / \mathrm{d}$ low molecular weight heparin calcium for 3 days in our hospital, 1 patient $(2.0 \%)$ was without vascular murmur and tremor. The rest were confirmed and treated as above. There was statistically a significant difference in the rate of internal fistula thrombosis between the control group and intervention group $(20.0 \%$ vs. $6.0 \%, p=0.037)$.

Aspirin is effective in reducing the formation of internal fistula thrombosis. This conclusion is consistent with previous research reports. ${ }^{4}$ The mechanism of aspirin inhibition of internal fistula thrombosis is related to its inhibition of platelet aggregation. ${ }^{5}$ No adverse reactions such as gastrointestinal bleeding were observed in this study, indicating that the dose of $100 \mathrm{mg} / \mathrm{d}$ aspirin was safe for uremic hemodialysis patients.

However, the sample size of this study was small and the clinical observation time was short. The adverse reactions of aspirin need further observation. Therefore, it should still be alert to the occurrence of gastrointestinal bleeding in clinical treatment. Thus it is recommended to regularly monitor the blood coagulation, fecal occult blood and other indicators during the use of aspirin treatment, in order to find abnormal situations and conduct timely treatment.

\section{REFERENCES}

1. Duque JC, Martinez L, Tabbara M, Dvorquez D, Mehandru SK, Asif $A$, et al. Arteriovenous fistula maturation in patients with permanent access created prior to or after hemodialysis initiation. J Vasc Access 2017; 18:185-91.

2. Siddiqui MA, Ashraff S, Carline T. Maturation of arteriovenous fistula: Analysis of key factors. Kidney Res Clin Pract 2017; 36:318-28.

3. Tayebi P, Kazemzadeh G, Banihashem A, Ravari H. Effect of low dose aspirin and dipyridamole on primary patency of arteriovenous grafts in hemodialysis patients: A randomized double-blind placebo-controlled trial. Electron Physician 2018; 10:6135-9. 
4. Hasegawa T, Elder SJ, Bragg-Gresham JL, Pisoni RL, Yamazaki S, Akizawa $\mathrm{T}$, et al. Consistent aspirin use associated with improved arteriovenous fistula survival among incident hemodialysis patients in the dialysis outcomes and practice patterns study. Clin J Am Soc Nephrol 2008; 3: 1373-8.

5. Pulcinelli FM, Pignatelli P, Celestini A, Riondino S, Gazzaniga PP, Violi F. Inhibition of platelet aggregation by aspirin progressively decreases in long-term treated patients. J Am Coll Cardiol 2004; 43:979-84.
Hui Liu and Huijun Lu

Department of Vascular Surgery, The Affiliated Wuxi People's Hospital of Nanjing Medical University, 214000-China

Correspondence: Dr. Huijun Lu, Department of Vascular Surgery, The Affiliated Wuxi People's Hospital of Nanjing Medical University, 214000, China

E-mail:hkkxv81@163.com

Received: October 22, 2018; Accepted: January 14, 2019 …… 